\title{
Association of $I L-10$ and TNF- $\alpha$ Polymorphisms with Dental Peri-Implant Disease Risk: A Meta-Analysis, Meta-Regression, and Trial Sequential Analysis
}

\author{
Ladan Jamshidy ${ }^{1}$, Santosh Kumar Tadakamadla ${ }^{2, *(\mathbb{D},}$, Parsia Choubsaz ${ }^{3}$, Masoud Sadeghi ${ }^{4}$ (D) \\ and Jyothi Tadakamadla ${ }^{5}$ \\ 1 Department of Prosthodontics, School of Dentistry, Kermanshah University of Medical Sciences, \\ Kermanshah 6713954658, Iran; ladanjamshidy@yahoo.com \\ 2 School of Medicine and Dentistry \& Menzies Health Institute Queensland, Griffith University, \\ Brisbane, QLD 4222, Australia \\ 3 Department of Prosthodontics, School of Dentistry, Shahid Beheshti University of Medical Sciences, \\ Tehran 1983963113, Iran; parsia.choubsaz@sbmu.ac.ir \\ 4 Medical Biology Research Center, Kermanshah University of Medical Sciences, \\ Kermanshah 6714415185, Iran; sadeghi_mbrc@yahoo.com \\ 5 School of Medicine and Dentistry, Griffith University, Brisbane, QLD 4222, Australia; \\ j.tadakamadla@griffith.edu.au \\ * Correspondence: santoshkumar.tadakamadla@griffithuni.edu.au
}

check for

updates

Citation: Jamshidy, L.; Tadakamadla S.K.; Choubsaz, P.; Sadeghi, M.;

Tadakamadla, J. Association of $I L-10$ and TNF- $\alpha$ Polymorphisms with Dental Peri-Implant Disease Risk: A Meta-Analysis, Meta-Regression, and Trial Sequential Analysis. Int. J. Environ. Res. Public Health 2021, 18 7697. https://doi.org/10.3390/ ijerph18147697

Academic Editor: Paul B. Tchounwou

Received: 16 June 2021

Accepted: 16 July 2021

Published: 20 July 2021

Publisher's Note: MDPI stays neutral with regard to jurisdictional claims in published maps and institutional affiliations.

Copyright: (c) 2021 by the authors. Licensee MDPI, Basel, Switzerland. This article is an open access article distributed under the terms and conditions of the Creative Commons Attribution (CC BY) license (https:// creativecommons.org/licenses/by/ $4.0 /)$
Abstract: Genetic susceptibility has been reported to be an important risk factor for peri-implant disease (PID). The aim of this meta-analysis was to assess the association between TNF- $\alpha$ and IL10 polymorphisms and PID susceptibility. The Web of Science, Cochrane Library, Scopus, and PubMed/Medline databases were searched for studies published until 12 April 2021. RevMan 5.3, CMA 2.0, SPSS 22.0, and trial sequential analysis software were used. Twelve studies were included in our analysis. The pooled ORs for the association of TNF- $\alpha(-308 \mathrm{G}>A), I L-10(-1082 A>G)$, $I L-10(-819 \mathrm{C}>\mathrm{T})$, and $I L-10(-592 \mathrm{~A}>\mathrm{C})$ polymorphisms were $1.12,0.93,1.35$, and 0.77 for allelic; $1.42,0.95,3.41$, and 0.34 for homozygous; $1.19,1.88,1.23$, and 0.49 for heterozygous, $1.53,1.12,1.41$, and 0.39 for recessive; and 1.16, 1.87, 2.65, and 0.75 for dominant models, respectively, with all the estimates being insignificant. The results showed an association between TNF- $\alpha(-308 G>A)$ polymorphism and the risk of PID in patients of Asian ethnicity (OR $=1.59 ; p=0.03)$. The present meta-analysis illustrated that TNF- $\alpha(-308 \mathrm{G}>A), I L-10(-1082 A>G), I L-10(-819 C>T)$, and IL-10 $(-592 A>C)$ polymorphisms were not associated with the risk of PID, whereas TNF- $\alpha(-308 G>A)$ polymorphism was associated with an elevated risk of PID in Asian patients.

Keywords: peri-implant disease; implant failure; peri-implantitis; bone loss; cytokine; polymorphism

\section{Introduction}

Despite the high survival rate and success of dental implants, it has long been known that osseointegrated implants may suffer from biological complications, collectively referred to as peri-implant disease (PID) [1]. PIDs are defined as inflammatory lesions of the tissue around the implant and include mucositis around the implant (inflammatory lesion confined to the mucosa around the implant) and peri-implantitis (an inflammatory lesion of the mucosa that affects the supporting bone with bone loss) [2]. A recent metaanalysis included peri-implantitis, implant failure, and marginal bone loss as PIDs [3]. A review study showed peri-implantitis in $28 \%$ and $\geq 56 \%$ of cases and in $12 \%$ and $43 \%$ of implant sites [4]. A systematic review suggested that the prevalence of peri-implantitis was approximately 22\% (range: 1-47\%) [5]. Another study found the prevalence of dental implant failures to be $11 \%$ in males and $9 \%$ in females; this prevalence was dependent on implant length, implant diameter, and bone quality [6]. Marginal bone loss ( $>0.5 \mathrm{~mm})$ 
at implants was also recognized in 30\% of cases and $16 \%$ of implant sites [7]. Evidence suggests that those who are aged more than 60 years, smokers, receiving head and neck radiation, postmenopausal, suffering from diabetes, and receiving hormone replacement therapy experienced significantly elevated implant failure in comparison with healthy patients [8].

Genetic susceptibility has been shown to be a significant risk factor for peri-implantitis, and there are numerous studies assessing this in different populations [9-11]. Gene polymorphisms refer to changes in DNA sequencing, such as the regulation of inflammatory mediators, primarily the gene promoter region, which can affect gene function and the progression of inflammatory diseases [12,13]. Polymorphisms of cytokines associated with the risk of PID, such as interleukin (IL)-1A [14], IL-1B [14,15], IL-6 [16,17], tumor necrosis factor-alpha $(T N F-\alpha)[17]$, and $I L-10[15,18]$ as an anti-inflammatory cytokine, could inhibit the production of proinflammatory cytokines and the induction of B lymphocyte proliferation as well as prevent the proliferation and activation of natural killer cells [19]. TNF- $\alpha$ is another anti-inflammatory cytokine that plays an important role in inflammatory processes [17]. The role of TNF- $\alpha$ in the destruction of bone around the implant has been suggested by researchers [20]. A meta-analysis [21] assessed the association of TNF- $\alpha$ $(-308 G>A)$ and IL-10 (-1082 A $>G)$ polymorphisms with the risk of implant failure by including two and three studies, respectively. Another meta-analysis [3] investigated the role of TNF- $\alpha(-308 G>A)$ polymorphism in PID using the data from six studies. Their results did not show any association between these polymorphisms and the risk of dental implant failure [21] and PID [3].

The aim of this study was to evaluate the association between TNF- $\alpha(-308 G>A)$, IL-10 $(-1082 A>G), I L-10(-819 C>T)$, and IL-10 $(-592 A>C)$ polymorphisms and PID susceptibility with more studies and the addition of two new polymorphisms (IL-10 $(-819 \mathrm{C}>\mathrm{T})$ and IL-10 $(-592 \mathrm{~A}>\mathrm{C}))$, meta-regression, and trial sequential analysis (TSA) compared to two previous meta-analyses.

\section{Materials and Methods}

\subsection{Design}

The preferred reporting items for systematic review and meta-analysis (PRISMA) guidelines were used to report this study [22]. The PICO (patient/population, intervention, comparison, and outcomes) question was as follows: Is there an association between IL-10 and TNF- $\alpha$ polymorphisms and the risk of PID in patients with dental implants?

\subsection{Literature Search Strategy}

The Web of Science, Cochrane Library, Scopus, and PubMed/Medline databases were searched for studies published until 12 April 2021 without any restrictions. The searched terms were ("dental implant" or "oral implant*" or "peri-implant disease" or "implant loss" or "implant failure" or "peri-implantitis" or "periimplant" or "implant bone loss" or "failing implant") and ("interleukin-10" or "IL-10" or "IL10" or "TNFA" or "TNF- $\alpha$ " or "TNF-alpha" or "TNFalpha" or "tumor necrosis factor-alpha" or "tumor necrosis factor alpha" or "TNF $\alpha$ " or "tumor necrosis factor- $\alpha$ ") and ("polymorphism" or "allele" or "genotype*" or "variant ${ }^{* \prime}$ or "SNP"). In addition, we manually checked the references of seminal articles related to the subject area to ensure that no potential articles were missed.

\subsection{Eligibility Criteria}

The studies were retrieved from the databases by one author (M.S.), and the duplicates and irrelevant studies were then excluded. The studies were considered relevant if they met the following eligibility criteria: (I) case-control design; (II) PID as the outcome of interest; (III) reporting TNF- $\alpha$ ( $-308 \mathrm{G}>A$ ), $I L-10(-1082 A>G), I L-10(-819 C>T)$, or IL-10 ( $-592 A>C$ ) polymorphisms with any genetic models; and (IV) having the required data to calculate the odds ratios (ORs) with $95 \%$ confidence intervals (CIs) for the genetic models. The studies were removed if they did not have the required data regarding 
genotype distributions or were animal studies, meta-analyses, review articles, letters to the editor, reported secondary data, and reported genotype distributions after treatment. The second author (L.J.) rechecked the relevant articles based on the eligibility criteria. Any disagreement between the two authors was resolved by discussion.

\subsection{Data Extraction}

One author (M.S.) independently extracted the data from each study and another author (J.T) rechecked them. The information retrieved from the studies included the first author's name, publication year, ethnic group, control source, mean/median age and male/female ratio in the two groups (patients and controls), genotyping method, form of disease, number of patients or controls, the $p$-value of Hardy-Weinberg equilibrium (HWE) in controls, the quality score, and the distribution of genotypes in the two groups. If there was a disagreement between the authors, the problem was resolved by a short discussion.

\subsection{Quality of Assessment}

One author (L.J) distinguished the quality of each included article using the modified Newcastle-Ottawa Quality Assessment Scale questionnaire, which was used in a similar meta-analysis involving gene polymorphisms. It involves assigning scores ranging from 0-2 and 0-1 on five (representativeness of cases, ascertainment of case outcomes, ascertainment of controls, $\mathrm{H}-\mathrm{W}$ equilibrium in controls, and association assessment) and two (description of follow-up and genotyping examination) criteria, respectively. A maximum total score of 12 was possible for each study [3].

\subsection{Statistical Analyses}

The Review Manager 5.3 (RevMan 5.3; the Cochrane Collaboration, the Nordic Cochrane Centre, Copenhagen, Denmark) was used to calculate crude odds ratio (OR) and $95 \%$ confidence interval (CI) showing the association between IL-10 and TNF- $\alpha$ polymorphisms and dental PID risk in the five genetic models. To evaluate the pooled OR significance, the $\mathrm{Z}$ test was applied with a $p<0.05$. The Cochrane $\mathrm{Q}$ test and $\mathrm{I}^{2}$ statistic showed the heterogeneity (inconsistency in the polymorphism effect across primary studies). If there was a statistically significant heterogeneity $\left(p<0.1\right.$ or $\left.\mathrm{I}^{2}>50 \%\right)$, we used a random-effect model (DerSimonian and Laird method) [23], and if there was no significant heterogeneity, a fixed-effect model (Mantel-Haenszel method) [24] was used.

The chi-square test was used to calculate the $p$-value of HWE in the control group of each study, with $p<0.05$ indicating a deviation from the HWE.

Subgroup, sensitivity, and meta-regression analyses were performed where possible depending on the number of studies available. The subgroup analysis for explanation of heterogeneity based on a priori hypothesis was done for TNF- $\alpha(-308 \mathrm{G}>A$ ) polymorphism according to the ethnicity, control source, disease form, and number of individuals.

The funnel plots were analyzed by the Egger's and Begg's tests (with $p$-values $<0.05$ indicating statistically significant existence of the publication bias). To evaluate the stability of the pooled results, we used sensitivity analyses ("one study removed" and "cumulative analysis") for TNF- $\alpha(-308 \mathrm{G}>A)$ and IL-10 (-819 C > T) polymorphisms. The Comprehensive Meta-Analysis version 2.0 (CMA 2.0; Biostat Inc., Englewood, NJ, USA) was used for sensitivity analyses and assessing publication bias. A meta-regression was performed to check the effect of publication year and number of individuals on the pooled results of TNF- $\alpha(-308 G>A)$ polymorphism. SPSS version 22.0 software (IBM Corp. Release 2013. IBM SPSS Statistics for Windows, Version 22.0. Armonk, NY: IBM Corp) was used to calculate the results of meta-regression.

Each meta-analysis may create a false-positive or -negative conclusion [25]. Hence, TSA was conducted using TSA software (version 0.9.5.10 beta) (Copenhagen Trial Unit, Centre for Clinical Intervention Research, Rigshospitalet, Copenhagen, Denmark) to reduce these statistical errors [26]. Additionally, a threshold of futility was tested by TSA to earn a conclusion of no effect before reaching the information size. The required information size 
(RIS) based on an alpha risk of $5 \%$, a beta risk of $20 \%$, and a two-sided boundary type was computed. For those analyses where the Z-curve reached the RIS line or monitored the boundary line or futility area, it was considered that the studies had adequate sample size and their results were valid. Otherwise, it was assumed that the available information was inadequate and more evidence was needed.

\section{Results}

\subsection{Study Selection}

Through the electronic and manual search, 63 records were identified (Figure 1). After removing the duplicates, 30 records were screened, while 10 irrelevant records were removed. A total of 20 full-text articles were evaluated for possible inclusion, and 8 of them were deemed irrelevant and excluded with reasons (one animal study, two reviews, one reported gingival crevicular fluid level of TNF- $\alpha$ and not polymorphisms, two meta-analyses, one systematic review, and one reported implant failure after total hip arthroplasty). Finally, 12 studies were included in our analysis.

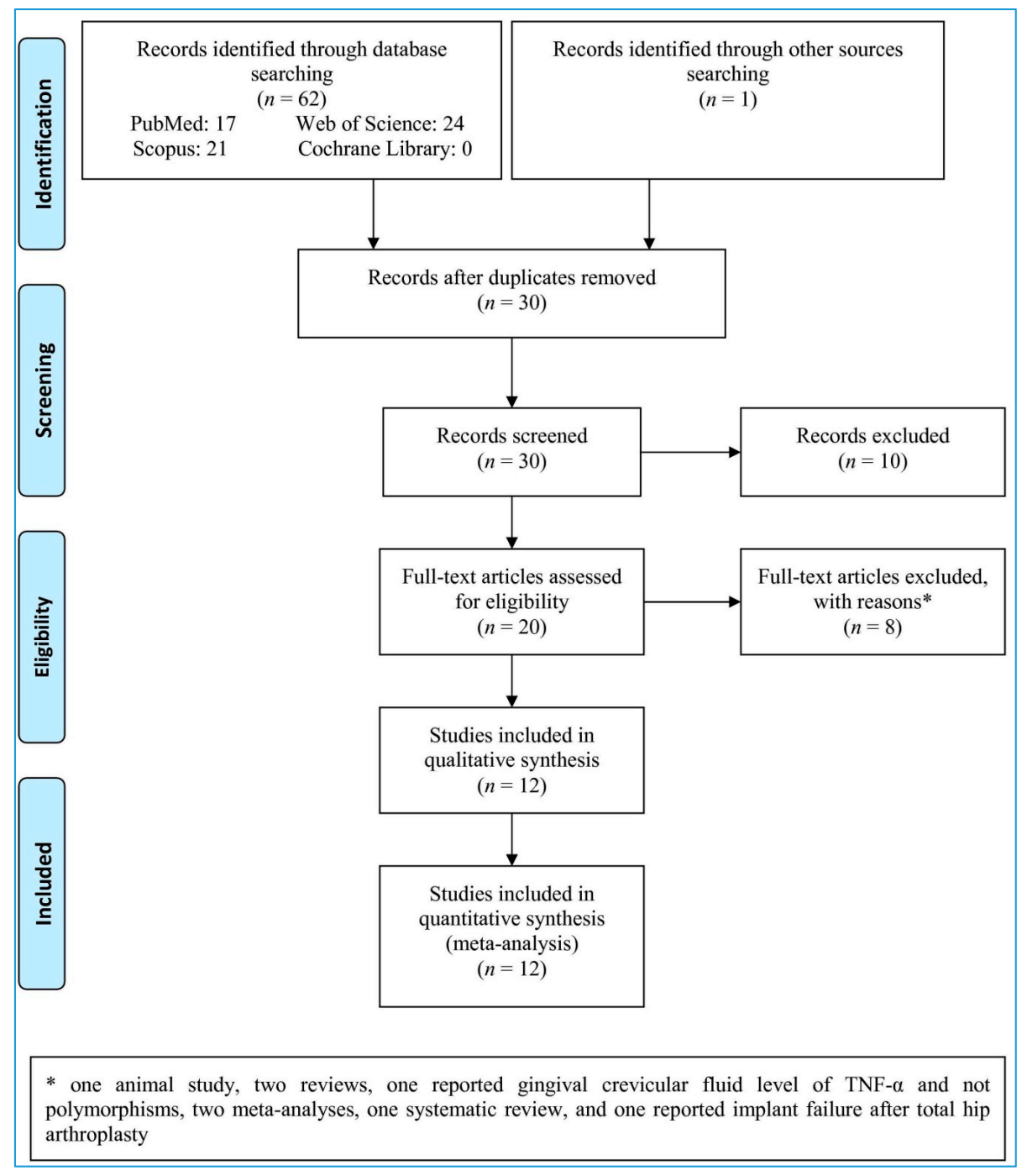

Figure 1. Flow chart of the study selection.

\subsection{Quality Assessment}

The quality score for the studies based on modified the Newcastle-Ottawa Scale (NOS) is shown in Table 1. The scores ranged from 8 to 10 . 
Table 1. Characteristics of studies included in the analysis.

\begin{tabular}{|c|c|c|c|c|c|c|c|c|c|c|c|c|}
\hline \multirow{2}{*}{$\begin{array}{c}\text { First Author, } \\
\text { Publication Year }\end{array}$} & \multirow[b]{2}{*}{ Country } & \multirow[b]{2}{*}{ Ethnicity } & \multirow{2}{*}{$\begin{array}{l}\text { Control } \\
\text { Source }\end{array}$} & \multicolumn{3}{|c|}{ Case } & \multicolumn{3}{|c|}{ Control } & \multirow{2}{*}{$\begin{array}{l}\text { Genotyping } \\
\text { Method }\end{array}$} & \multirow[b]{2}{*}{ Form of Disease } & \multirow{2}{*}{$\begin{array}{l}\text { Quality } \\
\text { Score }\end{array}$} \\
\hline & & & & Number & $\begin{array}{c}\text { Mean/Median } \\
\text { Age, Year }\end{array}$ & $\operatorname{Sex}(M / F)$ & Number & $\begin{array}{c}\text { Mean/Median } \\
\text { Age, Year }\end{array}$ & $\operatorname{Sex}(M / F)$ & & & \\
\hline Campos, 2004 [27] & Brazil & Mixed & HB & 28 & 52.7 & $13 / 15$ & 38 & 43.2 & $18 / 20$ & PCR & Implant failure & 10 \\
\hline $\mathrm{Lu}, 2009$ [29] & China & Asian & $\mathrm{HB}$ & 18 & 47 & $14 / 4$ & 26 & 48 & $15 / 11$ & PCR & $\begin{array}{l}\text { Marginal bone } \\
\text { loss }\end{array}$ & 8 \\
\hline Gurol, 2011 [30] & Turkey & Caucasian & PB & 16 & Range: 15-38 & - & 23 & Range: 15-38 & - & ARMS-PCR & Implant failure & 8 \\
\hline Pigossi, 2012 [18] & Brazil & Mixed & HB & 92 & 55.1 & $37 / 55$ & 185 & 53.1 & $64 / 121$ & RT-PCR & Implant failure & 8 \\
\hline $\begin{array}{c}\text { Jacobi-Gresser, } \\
2013 \text { [31] }\end{array}$ & Germany & Caucasian & $\mathrm{HB}$ & 41 & 51.1 & $18 / 23$ & 68 & 51.8 & $16 / 52$ & PCR & Implant failure & 8 \\
\hline Rakic, 2015 [32] & Serbia & Caucasian & НB & 180 & 53.2 & $102 / 78$ & 189 & 49.4 & $99 / 90$ & PCR-RFLP & Peri-implantitis & 10 \\
\hline $\begin{array}{l}\text { Petkovic-Curcin, } \\
2017 \text { [17] }\end{array}$ & Serbia & Caucasian & $\mathrm{HB}$ & 34 & 58 & $26 / 8$ & 64 & 58 & $44 / 20$ & PCR-RFLP & Peri-implantitis & 8 \\
\hline Ribeiro, 2017 [33] & Brazil & Mixed & НВ & 29 & Range: 21-80 & - & 61 & Range: $21-80$ & - & ARMS-PCR & Implant failure & 8 \\
\hline Broker, 2018 [34] & Brazil & Mixed & HB & 81 & 52.9 & $30 / 51$ & 163 & 51 & $52 / 111$ & RT-PCR & Implant failure & 9 \\
\hline He, 2020 [14] & China & Asian & PB & 144 & 45.1 & $88 / 56$ & 174 & 44.3 & $92 / 82$ & PCR & Peri-implantitis & 9 \\
\hline Saremi, 2021 [15] & Iran & Caucasian & PB & 50 & 42.2 & $24 / 26$ & 89 & 40.4 & $43 / 46$ & PCR-RFLP & Peri-implantitis & 9 \\
\hline
\end{tabular}

HB: hospital-based; PB: population-based; RT-PCR: real-time polymerase chain reaction; ARMS: amplification-refractory mutation system; RFLP: restriction fragment length polymorphism. 


\subsection{Study Characteristics}

Out of the 12 studies [14,15,17,18,27-34], five [15,17,30-32] were reported in Caucasian, five $[18,27,28,33,34]$ in mixed race, and two [14,29] in Asian ethnicities (Table 1). The control source was hospital-based in nine studies [17,18,27-29,31-34] and population-based in three studies $[14,15,30]$. The genotyping method in all studies was based on polymerase chain reaction (PCR). The form of PID in six [18,27,30,31,33,34], five [14,15,17,28,32], and one [29] studies were implant failure, peri-implantitis, and marginal bone loss, respectively.

Table 2 demonstrates the distribution of study population in the included studies based on the genotypes of TNF- $\alpha(-308 \mathrm{G}>A), I L-10(-1082 A>G), I L-10(-819 C>T)$, and IL-10 $(-592 A>C)$ polymorphisms. Ten $[14,15,17,27-32,34]$ studies reported genotype prevalence of TNF- $\alpha(-308 G>A)$, four $[17,18,30,33]$ reported $I L-10(-1082 A>G)$, three $[15,18,30]$ reported $I L-10(-819 C>T)$, and two $[15,18]$ reported $I L-10(-592 A>C)$ polymorphisms. Among the studies reporting TNF- $\alpha(-308 G>A)$ polymorphism, the control group in three studies $[14,30,32]$ had a deviation from HWE. Among the studies reporting $I L-$ $10(-819 C>T)$ polymorphism, one study [30] showed a deviation from HWE for the control group.

Table 2. Distribution of the genotypes of four polymorphisms.

\begin{tabular}{|c|c|c|c|c|c|c|c|}
\hline \multirow{3}{*}{ First Author, Publication Year } & \multicolumn{6}{|c|}{$T N F-\alpha(-308 G>A)$} & \multirow{3}{*}{$\begin{array}{c}p \text {-Value of HWE in } \\
\text { Control }\end{array}$} \\
\hline & \multicolumn{3}{|c|}{ Case } & \multicolumn{3}{|c|}{ Control } & \\
\hline & GG & GA & AA & GG & GA & AA & \\
\hline Campos, 2004 [27] & 26 & 2 & 0 & 32 & 6 & 0 & 0.597 \\
\hline Cury, 2009 [28] & 34 & 11 & 4 & 31 & 8 & 2 & 0.161 \\
\hline $\mathrm{Lu}, 2009[29]$ & 12 & 6 & 0 & 23 & 3 & 0 & 0.746 \\
\hline Gurol, 2011 [30] & 1 & 14 & 1 & 4 & 19 & 0 & $<0.001$ \\
\hline Jacobi-Gresser, 2013 [31] & 22 & 17 & 2 & 47 & 19 & 2 & 0.962 \\
\hline Rakic, 2015 [32] & 157 & 20 & 3 & 165 & 21 & 3 & 0.026 \\
\hline Petkovic-Curcin, 2017 [17] & 15 & & & 56 & & 8 & NA \\
\hline Broker, 2018 [34] & 63 & 16 & 0 & 128 & 32 & 2 & 1.000 \\
\hline $\mathrm{He}, 2020$ [14] & 113 & 11 & 20 & 146 & 12 & 16 & $<0.001$ \\
\hline \multirow[t]{3}{*}{ Saremi, 2021 [15] } & 4 & 12 & 34 & 4 & 18 & 67 & 0.074 \\
\hline & \multicolumn{6}{|c|}{$I L-10(-1082 A>G)$} & \\
\hline & AA & AG & GG & AA & AG & GG & \\
\hline Gurol, 2011 [30] & 2 & 9 & 4 & 3 & 15 & 4 & 0.086 \\
\hline Pigossi, 2012 [18] & 36 & 41 & 15 & 65 & 90 & 24 & 0.412 \\
\hline Petkovic-Curcin, 2017 [17] & 6 & & & 25 & & 39 & NA \\
\hline \multirow[t]{3}{*}{ Ribeiro, 2017 [33] } & 6 & 16 & 7 & 11 & 24 & 26 & 0.204 \\
\hline & \multicolumn{6}{|c|}{$I L-10(-819 C>T)$} & \\
\hline & $\mathrm{CC}$ & $\mathrm{CT}$ & $\mathrm{TT}$ & $\mathrm{CC}$ & $\mathrm{CT}$ & TT & \\
\hline Gurol, 2011 [30] & 0 & 12 & 1 & 1 & 19 & 1 & $<0.001$ \\
\hline Pigossi, 2012 [18] & 37 & 38 & 11 & 82 & 76 & 19 & 0.824 \\
\hline \multirow[t]{3}{*}{ Saremi, 2021 [15] } & 22 & 21 & 7 & 53 & 35 & 1 & 0.067 \\
\hline & \multicolumn{6}{|c|}{$I L-10(-592 A>C)$} & \\
\hline & AA & $\mathrm{AC}$ & $\mathrm{CC}$ & AA & $\mathrm{AC}$ & $\mathrm{CC}$ & \\
\hline Pigossi, 2012 [18] & 24 & 38 & 12 & 87 & 77 & 18 & 0.873 \\
\hline Saremi, 2021 [15] & 8 & 26 & 16 & 1 & 35 & 53 & 0.067 \\
\hline
\end{tabular}

\subsection{Pooled Analyses}

The results of meta-analyses based on five genetic models for TNF- $\alpha(-308 G>A)$ polymorphism are shown in Table 3. The pooled ORs were 1.12 (95\%CI: $0.90-1.39 ; p=0.32$; $\left.\mathrm{I}^{2}=43 \%\right), 1.42$ (95\%CI: 0.85-2.37; $\left.p=0.18 ; \mathrm{I}^{2}=0 \%\right), 1.19$ (95\%CI: $0.87-1.63 ; p=0.28 ; \mathrm{I}^{2}=0 \%$ ), 1.53 (95\%CI: 0.95-2.45; $p=0.08 ; \mathrm{I}^{2}=59 \%$ ), and 1.16 (95\%CI: $0.74-1.81 ; p=0.52 ; \mathrm{I}^{2}=0 \%$ ) 
for allelic, homozygous, heterozygous, recessive, and dominant models, respectively. The results showed that $T N F-\alpha(-308 G>A)$ polymorphism was not associated with PID risk.

Table 3. The results of meta-analyses based on five genetic models for TNF- $\alpha$ ( $-308 G>A)$ polymorphism.

\begin{tabular}{|c|c|c|c|c|c|c|c|}
\hline \multirow{2}{*}{ Genetic Model } & \multirow{2}{*}{$\begin{array}{l}\text { First Author, } \\
\text { Publication Year }\end{array}$} & \multicolumn{2}{|c|}{ Case } & \multicolumn{2}{|c|}{ Control } & \multirow{2}{*}{ Weight } & \multirow{2}{*}{$\begin{array}{c}\text { Odds Ratio } \\
\text { M-H, Fixed, } 95 \% \mathrm{CI}\end{array}$} \\
\hline & & Events & Total & Events & Total & & \\
\hline \multirow{9}{*}{ A vs. $G$} & Campos, 2004 [27] & 2 & 56 & 6 & 76 & $3.3 \%$ & $0.43[0.08,2.23]$ \\
\hline & Cury, 2009 [28] & 19 & 98 & 12 & 82 & $7.0 \%$ & $1.40[0.64,3.09]$ \\
\hline & $\mathrm{Lu}, 2009$ [29] & 6 & 36 & 3 & 52 & $1.4 \%$ & $3.27[0.76,14.04]$ \\
\hline & Gurol, 2011 [30] & 16 & 32 & 19 & 46 & $5.2 \%$ & $1.42[0.57,3.52]$ \\
\hline & Jacobi-Gresser, 2013 [31] & 21 & 82 & 23 & 136 & $8.5 \%$ & $1.69[0.87,3.30]$ \\
\hline & Rakic, 2015 [32] & 26 & 324 & 27 & 388 & $15.0 \%$ & $1.17[0.67,2.04]$ \\
\hline & Broker, 2018 [34] & 26 & 360 & 36 & 324 & $23.3 \%$ & $0.62[0.37,1.06]$ \\
\hline & $\mathrm{He}, 2020[14]$ & 51 & 288 & 44 & 348 & $21.8 \%$ & $1.49[0.96,2.30]$ \\
\hline & Saremi, 2021 [15] & 80 & 100 & 152 & 178 & $14.5 \%$ & $0.68[0.36,1.30]$ \\
\hline Subtotal $(95 \% \mathrm{CI})$ & & \multicolumn{3}{|c|}{1376} & 1630 & $100.0 \%$ & $1.12[0.90,1.39]$ \\
\hline Total events & & 247 & & 322 & & & \\
\hline \multicolumn{8}{|c|}{$\begin{array}{c}\text { Heterogeneity: } \mathrm{Chi}^{2}=14.03, \mathrm{df}=8(p=0.08) ; \mathrm{I}^{2}=43 \% \\
\text { Test for overall effect: } \mathrm{Z}=1.00(p=0.32)\end{array}$} \\
\hline \multirow{9}{*}{ AA vs. GG } & Campos, 2004 [27] & 0 & 26 & 0 & 32 & & Not estimable \\
\hline & Cury, 2009 [28] & 0 & 12 & 0 & 23 & & Not estimable \\
\hline & $\mathrm{Lu}, 2009[29]$ & 4 & 38 & 2 & 33 & $7.9 \%$ & $1.82[0.31,10.66]$ \\
\hline & Gurol, 2011 [30] & 1 & 2 & 0 & 4 & $0.8 \%$ & $9.00[0.22,362.48]$ \\
\hline & Jacobi-Gresser, 2013 [31] & 2 & 24 & 2 & 49 & $5.0 \%$ & $2.14[0.28,16.17]$ \\
\hline & Rakic, 2015 [32] & 3 & 160 & 3 & 168 & $11.8 \%$ & $1.05[0.21,5.28]$ \\
\hline & Broker, 2018 [34] & 0 & 63 & 2 & 130 & $6.7 \%$ & $0.40[0.02,8.56]$ \\
\hline & $\mathrm{He}, 2020$ [14] & 20 & 123 & 16 & 162 & $47.6 \%$ & $1.77[0.88,3.58]$ \\
\hline & Saremi, 2021 [15] & 34 & 38 & 67 & 71 & $20.2 \%$ & $0.51[0.12,2.15]$ \\
\hline Subtotal $(95 \% \mathrm{CI})$ & & \multicolumn{3}{|c|}{486} & 672 & $100.0 \%$ & $1.42[0.85,2.37]$ \\
\hline Total events & & 64 & & 92 & & & \\
\hline \multicolumn{8}{|c|}{$\begin{array}{l}\text { Heterogeneity: } \mathrm{Chi}^{2}=4.30, \mathrm{df}=6(p=0.64) ; \mathrm{I}^{2}=0 \% \\
\text { Test for overall effect: } \mathrm{Z}=1.33(p=0.18)\end{array}$} \\
\hline \multirow{9}{*}{ GA vs. GG } & Campos, 2004 [27] & 2 & 28 & 6 & 38 & $6.7 \%$ & $0.41[0.08,2.21]$ \\
\hline & Cury, 2009 [28] & 11 & 45 & 8 & 39 & $9.2 \%$ & $1.25[0.45,3.52]$ \\
\hline & $\mathrm{Lu}, 2009[29]$ & 6 & 18 & 3 & 26 & $2.3 \%$ & $3.83[0.81,18.09]$ \\
\hline & Gurol, 2011 [30] & 14 & 15 & 19 & 23 & $1.4 \%$ & $2.95[0.30,29.32]$ \\
\hline & Jacobi-Gresser, 2013 [31] & 17 & 39 & 19 & 66 & $11.3 \%$ & $1.91[0.84,4.37]$ \\
\hline & Rakic, 2015 [32] & 20 & 177 & 21 & 186 & $25.9 \%$ & $1.00[0.52,1.92]$ \\
\hline & Broker, 2018 [34] & 16 & 79 & 32 & 160 & $24.0 \%$ & $1.02[0.52,1.99]$ \\
\hline & $\mathrm{He}, 2020[14]$ & 11 & 124 & 12 & 158 & $13.7 \%$ & $1.18[0.50,2.78]$ \\
\hline & Saremi, 2021 [15] & 12 & 16 & 18 & 22 & $5.4 \%$ & $0.67[0.14,3.19]$ \\
\hline Subtotal (95\% CI) & & & 541 & & 718 & $100.0 \%$ & $1.19[0.87,1.63]$ \\
\hline Total events & & 109 & & 138 & & & \\
\hline \multicolumn{8}{|c|}{$\begin{array}{l}\text { Heterogeneity: } \mathrm{Chi}^{2}=6.60, \mathrm{df}=8(p=0.58) ; \mathrm{I}^{2}=0 \% \\
\text { Test for overall effect: } \mathrm{Z}=1.09(p=0.28)\end{array}$} \\
\hline \multirow{7}{*}{ AA + GA vs. GG } & Campos, 2004 [27] & 2 & 28 & 6 & 38 & $5.6 \%$ & $0.41[0.08,2.21]$ \\
\hline & Cury, 2009 [28] & 15 & 49 & 10 & 41 & $11.0 \%$ & $1.37[0.54,3.49]$ \\
\hline & Lu, 2009 [29] & 6 & 18 & 3 & 26 & $6.3 \%$ & $3.83[0.81,18.09]$ \\
\hline & Gurol, 2011 [30] & 15 & 16 & 19 & 23 & $3.5 \%$ & $3.16[0.32,31.29]$ \\
\hline & Jacobi-Gresser, 2013 [31] & 19 & 41 & 21 & 68 & $12.5 \%$ & $1.93[0.87,4.31]$ \\
\hline & Rakic, 2015 [32] & 23 & 180 & 24 & 189 & $14.6 \%$ & $1.01[0.55,1.86]$ \\
\hline & Petkovic-Curcin, 2017 [17] & 19 & 34 & 8 & 64 & $10.4 \%$ & $8.87[3.25,24.19]$ \\
\hline
\end{tabular}


Table 3. Cont.

\begin{tabular}{|c|c|c|c|c|c|c|c|}
\hline \multirow{2}{*}{ Genetic Model } & \multirow{2}{*}{$\begin{array}{c}\text { First Author, Publication } \\
\text { Year }\end{array}$} & \multicolumn{2}{|c|}{ Case } & \multicolumn{2}{|c|}{ Control } & \multirow{2}{*}{ Weight } & \multirow{2}{*}{$\begin{array}{c}\text { Odds Ratio } \\
\text { M-H, Fixed, } 95 \% \text { CI }\end{array}$} \\
\hline & & Events & Total & Events & Total & & \\
\hline & Broker, 2018 [34] & 16 & 79 & 34 & 162 & $14.0 \%$ & $0.96[0.49,1.86]$ \\
\hline & $\mathrm{He}, 2020$ [14] & 31 & 144 & 28 & 174 & $15.2 \%$ & $1.43[0.81,2.52]$ \\
\hline & Saremi, 2021 [15] & 46 & 50 & 85 & 89 & $7.0 \%$ & $0.54[0.13,2.26]$ \\
\hline \multicolumn{2}{|l|}{ Subtotal $(95 \% \mathrm{CI})$} & \multicolumn{3}{|c|}{639} & 874 & $100.0 \%$ & $1.53[0.95,2.45]$ \\
\hline Total events & & \multicolumn{2}{|l|}{192} & \multicolumn{2}{|l|}{238} & & \\
\hline \multicolumn{8}{|c|}{$\begin{array}{c}\text { Heterogeneity: } \mathrm{Tau}^{2}=0.30 ; \mathrm{Chi}^{2}=21.81, \mathrm{df}=9(p=0.009) ; \mathrm{I}^{2}=59 \% \\
\text { Test for overall effect: } \mathrm{Z}=1.75(p=0.08)\end{array}$} \\
\hline \multirow{9}{*}{ AA vs. GG + GA } & Campos, 2004 [27] & 0 & 28 & 0 & 38 & & Not estimable \\
\hline & Cury, 2009 [28] & 0 & 18 & 0 & 26 & & Not estimable \\
\hline & $\mathrm{Lu}, 2009$ [29] & 4 & 49 & 2 & 41 & $5.5 \%$ & $1.73[0.30,9.98]$ \\
\hline & Gurol, 2011 [30] & 1 & 16 & 0 & 23 & $1.0 \%$ & $4.55[0.17,118.99]$ \\
\hline & Jacobi-Gresser, 2013 [31] & 2 & 41 & 2 & 68 & $4.0 \%$ & $1.69[0.23,12.50]$ \\
\hline & Rakic, 2015 [32] & 3 & 180 & 3 & 189 & $7.9 \%$ & $1.05[0.21,5.28]$ \\
\hline & Broker, 2018 [34] & 0 & 79 & 2 & 162 & $4.5 \%$ & $0.40[0.02,8.51]$ \\
\hline & $\mathrm{He}, 2020$ [14] & 20 & 144 & 16 & 174 & $34.4 \%$ & $1.59[0.79,3.20]$ \\
\hline & Saremi, 2021 [15] & 34 & 50 & 67 & 89 & $42.6 \%$ & $0.70[0.32,1.50]$ \\
\hline Subtotal $(95 \% \mathrm{CI})$ & & \multicolumn{3}{|c|}{605} & 810 & $100.0 \%$ & $1.16[0.74,1.81]$ \\
\hline Total events & & 64 & & 92 & & & \\
\hline
\end{tabular}

Heterogeneity: $\mathrm{Chi}^{2}=3.98, \mathrm{df}=6(p=0.68) ; \mathrm{I}^{2}=0 \%$

Test for overall effect: $Z=0.64(p=0.52)$

The pooled ORs for allelic, homozygous, heterozygous, recessive, and dominant models of $I L-10(-1082 A>G)$ polymorphism were 0.93 (95\%CI: $0.69-1.25 ; p=0.61$; $\left.\mathrm{I}^{2}=0 \%\right), 0.95$ (95\%CI: 0.51-1.79; $\left.p=0.88 ; \mathrm{I}^{2}=0 \%\right), 1.88$ (95\%CI: 0.55-1.43; $p=0.62 ; \mathrm{I}^{2}=0 \%$ ), 1.12 (95\%CI: $0.74-1.68 ; p=0.60 ; \mathrm{I}^{2}=35 \%$ ), and 1.87 (95\%CI: $0.36-2.11 ; p=0.76 ; \mathrm{I}^{2}=56 \%$ ), respectively (Table 4$)$. The results showed that IL-10 $(-1082 A>G)$ polymorphism was not associated with susceptibility to PID.

Table 4. Results on the association of the five genetic models of $I L-10(-1082 \mathrm{~A}>\mathrm{G})$ polymorphism with the risk of PID.

\begin{tabular}{|c|c|c|c|c|c|c|c|}
\hline \multirow{2}{*}{ Genetic Model } & \multirow{2}{*}{$\begin{array}{l}\text { First Author, } \\
\text { Publication Year }\end{array}$} & \multicolumn{2}{|c|}{ Case } & \multicolumn{2}{|c|}{ Control } & \multirow{2}{*}{ Weight } & \multirow{2}{*}{$\begin{array}{c}\text { Odds Ratio } \\
\text { M-H, Fixed, } 95 \% \mathrm{CI}\end{array}$} \\
\hline & & Events & Total & Events & Total & & \\
\hline \multirow{3}{*}{ G vs. A } & Gurol, 2011 [30] & 17 & 30 & 23 & 44 & $9.1 \%$ & $1.19[0.47,3.04]$ \\
\hline & Pigossi, 2012 [18] & 71 & 184 & 138 & 358 & $64.5 \%$ & $1.00[0.70,1.44]$ \\
\hline & Ribeiro, 2017 [33] & 30 & 58 & 76 & 122 & $26.5 \%$ & $0.65[0.34,1.22]$ \\
\hline Subtotal $(95 \% \mathrm{CI})$ & & \multicolumn{3}{|c|}{272} & 524 & $100.0 \%$ & $0.93[0.69,1.25]$ \\
\hline Total events & & \multicolumn{2}{|l|}{118} & \multicolumn{2}{|l|}{237} & & \\
\hline \multicolumn{8}{|c|}{$\begin{array}{l}\text { Heterogeneity: } \mathrm{Chi}^{2}=1.68, \mathrm{df}=2(p=0.43) ; \mathrm{I}^{2}=0 \% \\
\text { Test for overall effect: } \mathrm{Z}=0.51(p=0.61)\end{array}$} \\
\hline \multirow{3}{*}{ GG vs. AA } & Gurol, 2011 [30] & 4 & 6 & 4 & 7 & $6.2 \%$ & $1.50[0.16,14.42]$ \\
\hline & Pigossi, 2012 [18] & 15 & 51 & 24 & 89 & $62.3 \%$ & $1.13[0.53,2.42]$ \\
\hline & Ribeiro, 2017 [33] & 7 & 13 & 26 & 37 & $31.5 \%$ & $0.49[0.13,1.81]$ \\
\hline Subtotal $(95 \% \mathrm{CI})$ & & & 70 & & 133 & $100.0 \%$ & $0.95[0.51,1.79]$ \\
\hline Total events & & 26 & & 54 & & & \\
\hline
\end{tabular}


Table 4. Cont.

\begin{tabular}{|c|c|c|c|c|c|c|c|}
\hline \multirow{2}{*}{ Genetic Model } & \multirow{2}{*}{$\begin{array}{l}\text { First Author, } \\
\text { Publication Year }\end{array}$} & \multicolumn{2}{|c|}{ Case } & \multicolumn{2}{|c|}{ Control } & \multirow{2}{*}{ Weight } & \multirow{2}{*}{$\begin{array}{c}\text { Odds Ratio } \\
\text { M-H, Fixed, } 95 \% \mathrm{CI}\end{array}$} \\
\hline & & Events & Total & Events & Total & & \\
\hline \multirow{3}{*}{ AG vs. AA } & Gurol, 2011 [30] & 9 & 11 & 15 & 18 & $5.9 \%$ & $0.90[0.13,6.46]$ \\
\hline & Pigossi, 2012 [18] & 41 & 77 & 90 & 155 & $79.7 \%$ & $0.82[0.47,1.43]$ \\
\hline & Ribeiro, 2017 [33] & 16 & 22 & 24 & 35 & $14.4 \%$ & $1.22[0.38,3.97]$ \\
\hline Subtotal $(95 \% \mathrm{CI})$ & & & 110 & & 208 & $100.0 \%$ & $0.88[0.55,1.43]$ \\
\hline Total events & & 66 & & 129 & & & \\
\hline \multicolumn{8}{|c|}{$\begin{array}{l}\text { Heterogeneity: } \mathrm{Chi}^{2}=0.36, \mathrm{df}=2(p=0.84) ; \mathrm{I}^{2}=0 \% \\
\text { Test for overall effect: } \mathrm{Z}=0.50(p=0.62)\end{array}$} \\
\hline \multirow{4}{*}{ GG + AG vs. AA } & Gurol, 2011 [30] & 13 & 15 & 19 & 22 & $4.7 \%$ & $1.03[0.15,7.02]$ \\
\hline & Pigossi, 2012 [18] & 56 & 92 & 114 & 179 & $69.2 \%$ & $0.89[0.53,1.49]$ \\
\hline & Petkovic-Curcin, 2017 [17] & 23 & 29 & 50 & 61 & $15.2 \%$ & $0.84[0.28,2.56]$ \\
\hline & Ribeiro, 2017 [33] & 28 & 34 & 39 & 64 & $10.9 \%$ & $2.99[1.08,8.25]$ \\
\hline Subtotal $(95 \% \mathrm{CI})$ & & & 170 & & 326 & $100.0 \%$ & $1.12[0.74,1.68]$ \\
\hline Total events & & 120 & & 222 & & & \\
\hline \multicolumn{8}{|c|}{$\begin{aligned} & \text { Heterogeneity: } \mathrm{Chi}^{2}=4.64, \mathrm{df}=3(p=0.20) ; \mathrm{I}^{2}=35 \% \\
& \text { Test for overall effect: } \mathrm{Z}=0.53(p=0.60)\end{aligned}$} \\
\hline \multirow{3}{*}{ GG vs. AA + AG } & Gurol, 2011 [30] & 4 & 15 & 4 & 22 & $20.8 \%$ & $1.64[0.34,7.91]$ \\
\hline & Pigossi, 2012 [18] & 15 & 92 & 24 & 179 & $44.1 \%$ & $1.26[0.62,2.54]$ \\
\hline & Ribeiro, 2017 [33] & 7 & 34 & 26 & 64 & $35.2 \%$ & $0.38[0.14,1.00]$ \\
\hline Subtotal $(95 \% \mathrm{CI})$ & & & 141 & & 265 & $100.0 \%$ & $0.87[0.36,2.11]$ \\
\hline Total events & & 26 & & 54 & & & \\
\hline
\end{tabular}

Heterogeneity: $\mathrm{Tau}^{2}=0.33 ; \mathrm{Chi}^{2}=4.50, \mathrm{df}=2(p=0.11) ; \mathrm{I}^{2}=56 \%$

Test for overall effect: $Z=0.31(p=0.76)$

For allelic, homozygous, heterozygous, recessive, and dominant models of IL-10 $(-819 \mathrm{C}>\mathrm{T})$ polymorphism, the pooled ORs were 1.35 (95\%CI: $\left.1.00-1.82 ; p=0.05 ; \mathrm{I}^{2}=37 \%\right)$, 3.41 (95\%CI: 0.52-22.17; $p=0.20 ; \mathrm{I}^{2}=60 \%$ ), 1.23 (95\%CI: $0.80-1.90 ; p=0.35 ; \mathrm{I}^{2}=0 \%$ ), 1.41 (95\%CI: 0.93-2.13; $p=0.10 ; \mathrm{I}^{2}=0 \%$ ), and 2.65 (95\%CI: 0.53-13.34; $p=0.24 ; \mathrm{I}^{2}=57 \%$ ), respectively (Table 5). The results reported that there was no association between $I L-10$ $(-819 \mathrm{C}>\mathrm{T})$ polymorphism and susceptibility to PID.

Table 5. Meta-analyses of studies involving five genetic models of $I L-10$ ( $-819 C>T$ ) polymorphism and the risk of PID.

\begin{tabular}{|c|c|c|c|c|c|c|c|}
\hline \multirow{2}{*}{ Genetic Model } & \multirow{2}{*}{$\begin{array}{l}\text { First Author, } \\
\text { Publication Year }\end{array}$} & \multicolumn{2}{|c|}{ Case } & \multicolumn{2}{|c|}{ Control } & \multirow{2}{*}{ Weight } & \multirow{2}{*}{$\begin{array}{c}\text { Odds Ratio } \\
\text { M-H, Fixed, } 95 \% \mathrm{CI}\end{array}$} \\
\hline & & Events & Total & Events & Total & & \\
\hline \multirow{3}{*}{ T vs. C } & Gurol, 2011 [30] & 14 & 26 & 21 & 42 & $10.1 \%$ & $1.17[0.44,3.11]$ \\
\hline & Pigossi, 2012 [18] & 60 & 172 & 114 & 354 & $66.3 \%$ & $1.13[0.77,1.66]$ \\
\hline & Saremi, 2021 [15] & 35 & 100 & 37 & 178 & $23.6 \%$ & $2.05[1.19,3.55]$ \\
\hline Subtotal $(95 \% \mathrm{CI})$ & & \multicolumn{3}{|c|}{298} & 574 & $100.0 \%$ & $1.35[1.00,1.82]$ \\
\hline Total events & & \multicolumn{2}{|l|}{109} & \multicolumn{2}{|l|}{172} & & \\
\hline \multicolumn{8}{|c|}{$\begin{aligned} \text { Heterogeneity: } \mathrm{Chi}^{2}=3.17, \mathrm{df} & =2(p=0.20) ; \mathrm{I}^{2}=37 \% \\
\text { Test for overall effect: } \mathrm{Z} & =1.97(p=0.05)\end{aligned}$} \\
\hline \multirow{3}{*}{ TT vs. CC } & Gurol, 2011 [30] & 1 & 1 & 1 & 2 & $16.3 \%$ & $3.00[0.06,151.19]$ \\
\hline & Pigossi, 2012 [18] & 11 & 48 & 19 & 101 & $51.2 \%$ & $1.28[0.56,2.97]$ \\
\hline & Saremi, 2021 [15] & 7 & 29 & 1 & 54 & $32.5 \%$ & $16.86[1.96,145.27]$ \\
\hline Subtotal $(95 \% \mathrm{CI})$ & & & 78 & & 157 & $100.0 \%$ & $3.41[0.52,22.17]$ \\
\hline Total events & & 19 & & 21 & & & \\
\hline
\end{tabular}


Table 5. Cont.

\begin{tabular}{|c|c|c|c|c|c|c|c|}
\hline \multirow{2}{*}{ Genetic Model } & \multirow{2}{*}{$\begin{array}{l}\text { First Author, } \\
\text { Publication Year }\end{array}$} & \multicolumn{2}{|c|}{ Case } & \multicolumn{2}{|c|}{ Control } & \multirow{2}{*}{ Weight } & \multirow{2}{*}{$\begin{array}{c}\text { Odds Ratio } \\
\text { M-H, Fixed, } 95 \% \mathrm{Cl}\end{array}$} \\
\hline & & Events & Total & Events & Total & & \\
\hline \multicolumn{8}{|c|}{$\begin{array}{c}\text { Heterogeneity: } \mathrm{Tau}^{2}=1.60 ; \mathrm{Chi}^{2}=4.99, \mathrm{df}=2(p=0.08) ; \mathrm{I}^{2}=60 \% \\
\text { Test for overall effect: } \mathrm{Z}=1.28(p=0.20)\end{array}$} \\
\hline \multirow{3}{*}{ CT vs. TT } & Gurol, 2011 [30] & 12 & 12 & 19 & 20 & $1.6 \%$ & $1.92[0.07,51.03]$ \\
\hline & Pigossi, 2012 [18] & 38 & 75 & 76 & 158 & $66.2 \%$ & $1.11[0.64,1.92]$ \\
\hline & Saremi, 2021 [15] & 21 & 43 & 35 & 88 & $32.2 \%$ & $1.45[0.69,3.01]$ \\
\hline Subtotal (95\% CI) & & & 130 & & 266 & $100.0 \%$ & $1.23[0.80,1.90]$ \\
\hline Total events & & 71 & & 130 & & & \\
\hline \multicolumn{8}{|c|}{$\begin{array}{l}\text { Heterogeneity: } \mathrm{Chi}^{2}=0.40, \mathrm{df}=2(p=0.82) ; \mathrm{I}^{2}=0 \% \\
\text { Test for overall effect: } \mathrm{Z}=0.93(p=0.35)\end{array}$} \\
\hline \multirow{3}{*}{$\mathrm{TT}+\mathrm{CT}$ vs. CC } & Gurol, 2011 [30] & 13 & 13 & 20 & 21 & $1.5 \%$ & $1.98[0.07,52.16]$ \\
\hline & Pigossi, 2012 [18] & 49 & 86 & 95 & 177 & $70.0 \%$ & $1.14[0.68,1.92]$ \\
\hline & Saremi, 2021 [15] & 29 & 50 & 36 & 89 & $28.5 \%$ & $2.03[1.01,4.11]$ \\
\hline Subtotal $(95 \% \mathrm{CI})$ & & & 149 & & 287 & $100.0 \%$ & $1.41[0.93,2.13]$ \\
\hline Total events & & 91 & & 151 & & & \\
\hline \multicolumn{8}{|c|}{$\begin{array}{l}\text { Heterogeneity: } \mathrm{Chi}^{2}=1.71, \mathrm{df}=2(p=0.43) ; \mathrm{I}^{2}=0 \% \\
\text { Test for overall effect: } \mathrm{Z}=1.63(p=0.10)\end{array}$} \\
\hline \multirow{3}{*}{ TT vs. $\mathrm{CC}+\mathrm{CT}$} & Gurol, 2011 [30] & 1 & 13 & 1 & 21 & $20.5 \%$ & $1.67[0.10,29.18]$ \\
\hline & Pigossi, 2012 [18] & 11 & 86 & 19 & 177 & $50.6 \%$ & $1.22[0.55,2.69]$ \\
\hline & Saremi, 2021 [15] & 7 & 50 & 1 & 89 & $28.9 \%$ & $14.33[1.71,120.16]$ \\
\hline Subtotal $(95 \% \mathrm{CI})$ & & & 149 & & 287 & $100.0 \%$ & $2.65[0.53,13.34]$ \\
\hline Total events & & 19 & & 21 & & & \\
\hline
\end{tabular}

Heterogeneity: $\mathrm{Tau}^{2}=1.18 ; \mathrm{Chi}^{2}=4.69, \mathrm{df}=2(p=0.10) ; \mathrm{I}^{2}=57 \%$

Test for overall effect: $\mathrm{Z}=1.18(p=0.24)$

Table 6 demonstrates the results for $I L-10(-592 A>C)$ polymorphism with data from two studies for $\mathrm{C}$ vs. A, CC vs. AA, AC vs. AA, CC + AC vs. AA, and CC vs. AA + AC genetic models. High heterogeneity was observed in all the models, the pooled ORs were 0.77 (95\%CI: 0.18-3.31; $p=0.73$ ), 0.34 (95\%CI: 0.00-23.53; $p=0.62$ ), 0.49 (95\%CI: $0.03-9.22$; $p=0.63$ ), 0.39 (95\%CI: 0.01-12.59; $p=0.60$ ), and 0.75 (95\%CI: $0.14-3.98 ; p=0.73$ ) for $\mathrm{C}$ vs. $\mathrm{A}, \mathrm{CC}$ vs. $\mathrm{AA}, \mathrm{AC}$ vs. $\mathrm{AA}, \mathrm{CC}+\mathrm{AC}$ vs. $\mathrm{AA}$, and $\mathrm{CC}$ vs. $\mathrm{AA}+\mathrm{AC}$, respectively. The results showed that there was no association between $I L-10(-592 A>C)$ polymorphism and susceptibility to PID.

Table 6. Meta-analyses of association between $I L-10(-592 A>C)$ polymorphism and PID risk based on five genetic models.

\begin{tabular}{|c|c|c|c|c|c|c|c|}
\hline \multirow{2}{*}{ Genetic Model } & \multirow{2}{*}{$\begin{array}{l}\text { First Author, } \\
\text { Publication Year }\end{array}$} & \multicolumn{2}{|c|}{ Case } & \multicolumn{2}{|c|}{ Control } & \multirow{2}{*}{ Weight } & \multirow{2}{*}{$\begin{array}{c}\text { Odds Ratio } \\
\text { M-H, Random, } 95 \% \text { CI }\end{array}$} \\
\hline & & Events & Total & Events & Total & & \\
\hline \multirow{2}{*}{ C vs. A } & Pigossi, 2012 [18] & 62 & 148 & 113 & 364 & $50.8 \%$ & $1.60[1.08,2.38]$ \\
\hline & Saremi, 2021 [15] & 58 & 100 & 141 & 178 & $49.2 \%$ & $0.36[0.21,0.62]$ \\
\hline Subtotal $(95 \% \mathrm{CI})$ & & \multicolumn{3}{|c|}{248} & 542 & $100.0 \%$ & $0.77[0.18,3.31]$ \\
\hline Total events & & \multicolumn{2}{|l|}{120} & \multicolumn{2}{|l|}{254} & & \\
\hline \multicolumn{8}{|c|}{$\begin{array}{c}\text { Heterogeneity: } \mathrm{Tau}^{2}=1.05 ; \mathrm{Chi}^{2}=19.07, \mathrm{df}=1(p<0.0001) ; \mathrm{I}^{2}=95 \% \\
\text { Test for overall effect: } \mathrm{Z}=0.35(p=0.73)\end{array}$} \\
\hline \multirow{2}{*}{ CC vs. AA } & Pigossi, 2012 [18] & 12 & 36 & 18 & 105 & $52.7 \%$ & $2.42[1.02,5.70]$ \\
\hline & Saremi, 2021 [15] & 16 & 24 & 53 & 54 & $47.3 \%$ & $0.04[0.00,0.32]$ \\
\hline
\end{tabular}


Table 6. Cont.

\begin{tabular}{|c|c|c|c|c|c|c|c|}
\hline \multirow{2}{*}{ Genetic Model } & \multirow{2}{*}{$\begin{array}{c}\text { First Author, } \\
\text { Publication Year }\end{array}$} & \multicolumn{2}{|c|}{ Case } & \multicolumn{2}{|c|}{ Control } & \multirow{2}{*}{ Weight } & \multirow{2}{*}{$\begin{array}{c}\text { Odds Ratio } \\
\text { M-H, Random, } 95 \% \mathrm{CI}\end{array}$} \\
\hline & & Events & Total & Events & Total & & \\
\hline Subtotal $(95 \% \mathrm{CI})$ & & & 60 & & 159 & $100.0 \%$ & $0.34[0.00,23.53]$ \\
\hline Total events & & 28 & & 71 & & & \\
\hline
\end{tabular}

Heterogeneity: $\mathrm{Tau}^{2}=8.70 ; \mathrm{Chi}^{2}=13.45, \mathrm{df}=1(p=0.0002) ; \mathrm{I}^{2}=93 \%$

Test for overall effect: $Z=0.50(p=0.62)$

\begin{tabular}{cccccccc}
\hline \multirow{2}{*}{ AC vs. AA } & Pigossi, 2012 [18] & 38 & 62 & 77 & 164 & $56.0 \%$ & $1.79[0.99,3.25]$ \\
& Saremi, 2021 [15] & 26 & 34 & 35 & 36 & $44.0 \%$ & $0.09[0.01,0.79]$ \\
\hline Subtotal (95\% CI) & & & 96 & & 200 & $100.0 \%$ & $0.49[0.03,9.22]$ \\
\hline Total events & & 64 & & 112 & & & \\
\hline
\end{tabular}

Heterogeneity: $\mathrm{Tau}^{2}=3.93 ; \mathrm{Chi}^{2}=7.11, \mathrm{df}=1(p=0.008) ; \mathrm{I}^{2}=86 \%$

Test for overall effect: $Z=0.48(p=0.63)$

\begin{tabular}{cccccccc}
\hline CC + AC vs. AA & Pigossi, 2012 [18] & 50 & 74 & 95 & 182 & $54.3 \%$ & $1.91[1.08,3.36]$ \\
& Saremi, 2021 [15] & 42 & 50 & 88 & 89 & $45.7 \%$ & $0.06[0.01,0.49]$ \\
\hline Subtotal $(95 \% \mathrm{CI})$ & & & 124 & & 271 & $100.0 \%$ & $0.39[0.01,12.59]$ \\
\hline Total events & & 92 & & 183 & & & \\
\hline
\end{tabular}

Heterogeneity: $\mathrm{Tau}^{2}=5.70 ; \mathrm{Chi}^{2}=10.16, \mathrm{df}=1(p=0.001) ; \mathrm{I}^{2}=90 \%$

Test for overall effect: $Z=0.53(p=0.60)$

\begin{tabular}{cccccccc}
\hline \multirow{2}{*}{ CC vs. AA + AC } & Pigossi, 2012 [18] & 12 & 74 & 18 & 182 & $49.6 \%$ & $1.76[0.80,3.87]$ \\
& Saremi, 2021 [15] & 16 & 50 & 53 & 89 & $50.4 \%$ & $0.32[0.15,0.66]$ \\
\hline Subtotal (95\%CI) & & & 124 & & 271 & $100.0 \%$ & $0.75[0.14,3.98]$ \\
\hline Total events & & 28 & & 71 & & & \\
\hline
\end{tabular}

Heterogeneity: $\mathrm{Tau}^{2}=1.31 ; \mathrm{Chi}^{2}=9.75, \mathrm{df}=1(p=0.002) ; \mathrm{I}^{2}=90 \%$

Test for overall effect: $Z=0.34(p=0.73)$

\subsection{Subgroup Analysis}

Subgroup analyses based on ethnicity, control source, disease form, and number of individuals were performed on the association between TNF- $\alpha(-308 \mathrm{G}>A$ ) polymorphism and PID risk (Table 7). The results showed that ethnicity was the only significant factor. Asian patients with TNF- $\alpha(-308 \mathrm{G}>A)$ polymorphism had a significant elevated risk of PID than the controls ( $\mathrm{OR}=1.59 ; p=0.03$ ), whereas there was no significant association between the polymorphism and PID risk for Caucasian and mixed ethnicities.

Table 7. Subgroup analyses based on ethnicity, control source, disease form, and sample size for five genetic models of TNF- $\alpha(-308 G>A$ ) polymorphism.

\begin{tabular}{|c|c|c|c|c|c|}
\hline \multirow{2}{*}{ Variable $\left(\mathbf{N}, \mathbf{N}^{\prime}\right)$} & A vs. G & AA vs. GG & GA vs. GG & AA + GA vs. GG & AA vs. $G G$ + GA \\
\hline & OR $(95 \% C I), p, I^{2}$ & OR $(95 \% \mathrm{CI}), p, \mathrm{I}^{2}$ & OR $(95 \% \mathrm{CI}), p, \mathrm{I}^{2}$ & OR $(95 \% \mathrm{CI}), p, \mathrm{I}^{2}$ & OR $(95 \% \mathrm{CI}), p, \mathrm{I}^{2}$ \\
\hline All $(9,10)$ & $\begin{array}{c}1.12(0.90,1.39) \\
0.32,43 \%\end{array}$ & $\begin{array}{c}1.42(0.85,2.37) \\
0.18,0 \%\end{array}$ & $\begin{array}{c}1.19(0.87,1.63) \\
0.28,0 \%\end{array}$ & $\begin{array}{c}1.53(0.95,2.45) \\
0.08,59 \%\end{array}$ & $\begin{array}{c}1.16(0.74,1.81) \\
0.52,0 \%\end{array}$ \\
\hline \multicolumn{6}{|l|}{ Ethnicity } \\
\hline Caucasian $(4,5)$ & $\begin{array}{c}1.14(0.82,1.59) \\
0.44,25 \%\end{array}$ & $\begin{array}{c}1.06(0.43,2.62) \\
0.89,0 \%\end{array}$ & $\begin{array}{c}1.26(0.79,2.01) \\
0.34,0 \%\end{array}$ & $\begin{array}{c}1.92(0.76,4.89) \\
0.17,75 \%\end{array}$ & $\begin{array}{c}0.89(0.47,1.68) \\
0.72,0 \%\end{array}$ \\
\hline Asian $(2,2)$ & $\begin{array}{c}1.59(1.05,2.42) \\
0.03,3 \% *\end{array}$ & $\begin{array}{c}1.77(0.88,3.58) \\
0.11\end{array}$ & $\begin{array}{c}1.57(0.75,3.27) \\
0.23,41 \%\end{array}$ & $\begin{array}{c}1.61(0.95,2.74) \\
0.08,27 \%\end{array}$ & $\begin{array}{c}1.59(0.79,3.20) \\
0.19\end{array}$ \\
\hline Mixed $(3,3)$ & $\begin{array}{c}0.77(0.51,1.16) \\
0.21,40 \%\end{array}$ & $\begin{array}{c}1.17(0.29,4.81) \\
0.83,0 \%\end{array}$ & $\begin{array}{c}0.97(0.57,1.64) \\
0.91,0 \%\end{array}$ & $\begin{array}{c}0.97(0.59,1.62) \\
0.92,0 \%\end{array}$ & $\begin{array}{c}1.14(0.28,4.63) \\
0.86,0 \%\end{array}$ \\
\hline Control source & & & & & \\
\hline
\end{tabular}


Table 7. Cont.

\subsection{Sensitivity Analysis}

Sensitivity analyses were performed by removing studies with a deviation of HWE in their controls for both TNF- $\alpha(-308 \mathrm{G}>\mathrm{A})$ and IL-10 $(-819 \mathrm{C}>\mathrm{T})$ polymorphisms (Table 8). In addition, "one study removed" and "cumulative analyses" were performed, and the results did not change for both the polymorphisms.

Table 8. Sensitivity analyses removing the studies with a deviation of HWE in their controls for TNF- $\alpha$ ( -308 G $>A)$ and IL-10 (-819 C > T) polymorphisms.

\begin{tabular}{|c|c|c|c|c|c|}
\hline \multirow{2}{*}{ Polymorphism (N, N') } & Allelic & Homozygous & Heterozygous & Recessive & Dominant \\
\hline & OR $(95 \% C I), p, I^{2}$ & OR $(95 \% C I), p, I^{2}$ & OR $(95 \% C I), p, I^{2}$ & OR $(95 \% \mathrm{CI}), p, \mathrm{I}^{2}$ & OR $(95 \% C I), p, I^{2}$ \\
\hline $\begin{array}{c}T N F-\alpha(-308 G>A) \\
(6,7)\end{array}$ & $\begin{array}{c}1.02(0.62,1.66) \\
0.95,54 \%\end{array}$ & $\begin{array}{c}0.95(0.39,2.35) \\
0.92,0 \%\end{array}$ & $\begin{array}{c}1.24(0.82,1.85) \\
0.31,12 \%\end{array}$ & $\begin{array}{c}1.61(0.78,3.32) \\
0.20,70 \%\end{array}$ & $\begin{array}{c}0.84(0.45,1.60) \\
0.60,0 \%\end{array}$ \\
\hline $\boldsymbol{I L} \mathbf{- 1 0}(-819 \mathrm{C}>\mathrm{T})(2)$ & $\begin{array}{c}1.47(0.82,2.64) \\
0.19,67 \%\end{array}$ & $\begin{array}{c}3.84(0.30,48.54) \\
0.30,80 \%\end{array}$ & $\begin{array}{c}1.22(0.78,1.89) \\
0.38,0 \%\end{array}$ & $\begin{array}{c}1.40(0.92,2.12) \\
0.11,40 \%\end{array}$ & $\begin{array}{c}3.43(0.30,38.86) \\
0.32,79 \%\end{array}$ \\
\hline
\end{tabular}

\subsection{Meta-Regression}

To check the effect of publication year and sample size on the pooled results of TNF- $\alpha$ ( $-308 \mathrm{G}>$ A) polymorphism, meta-regression was conducted. The findings demonstrated that the publication year and sample size were not confounding factors on the association between TNF- $\alpha$ ( $-308 G>A$ ) polymorphism and susceptibility to PID (Table 9).

Table 9. Meta-regression for TNF- $\alpha(-308 \mathrm{G}>A$ ) polymorphism based on publication year and sample size.

\begin{tabular}{|c|c|c|c|c|c|c|}
\hline Variable & & A vs. $G$ & AA vs. GG & GA vs. GG & $\begin{array}{c}\text { AA + GA vs. } \\
\text { GG }\end{array}$ & $\begin{array}{c}\text { AA vs. GG + } \\
\text { GA }\end{array}$ \\
\hline \multirow{3}{*}{$\begin{array}{c}\text { Year of } \\
\text { publication }\end{array}$} & $\mathrm{R}$ & 0.211 & 0.522 & 0.272 & 0.075 & 0.585 \\
\hline & Adjusted $\mathrm{R}^{2}$ & -0.092 & 0.127 & -0.058 & -0.119 & 0.210 \\
\hline & $p$-value & 0.586 & 0.229 & 0.479 & 0.837 & 0.168 \\
\hline \multirow{3}{*}{$\begin{array}{l}\text { Number of } \\
\text { individuals }\end{array}$} & $\mathrm{R}$ & 0.272 & 0.558 & 0.472 & 0.337 & 0.566 \\
\hline & Adjusted $\mathrm{R}^{2}$ & -0.058 & 0.173 & 0.112 & 0.003 & 0.185 \\
\hline & $p$-value & 0.479 & 0.193 & 0.200 & 0.341 & 0.185 \\
\hline
\end{tabular}




\subsection{Trial Sequential Analysis}

For TNF- $\alpha(-308 \mathrm{G}>A)$ and IL-10 $(-1082 A>G)$ polymorphisms, the Z-curve did not reach the RIS line or cross the boundary line or enter futility area, establishing that the evidence was not enough for significant results and more information was needed. With regard to IL-10 $(-819 \mathrm{C}>\mathrm{T})$ polymorphism, the Z-curve exceeded the RIS line, confirming that there was enough evidence to conclude that that the $I L-10(-819 C>T)$ polymorphism was not associated with the PID risk (Figure 2).

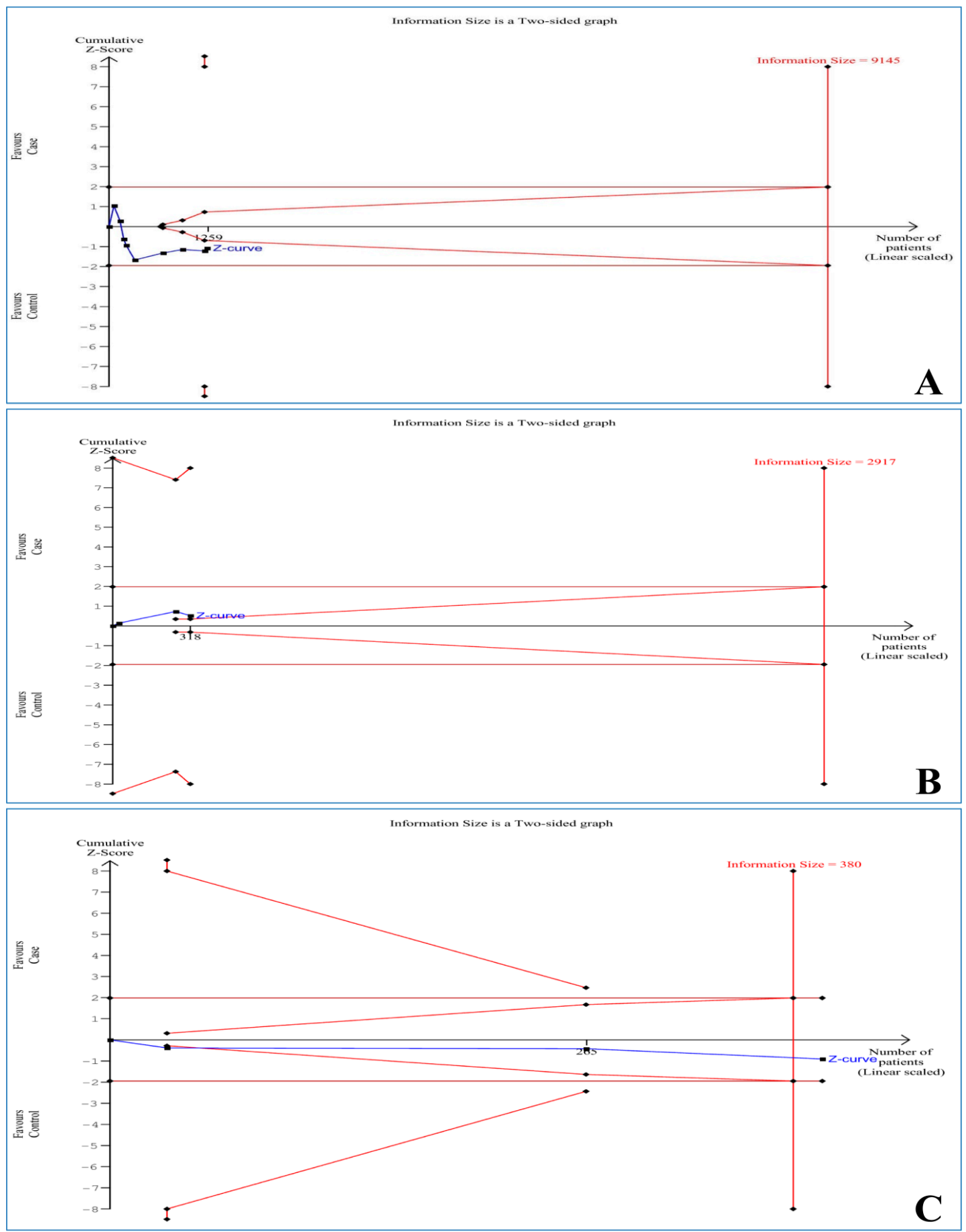

Figure 2. Trial sequential analysis for the association between polymorphisms and dental peri-implant disease risk based on heterozygous model: (A) TNF- $\alpha(-308 \mathrm{G}>\mathrm{A})$, (B) IL-10 (-1082 A > G), and (C) IL-10 (-819 C > T). 


\subsection{Publication Bias}

Funnel plots (Figure 3) along with Egger's and Begg's tests demonstrated that there was no publication bias for allelic (Egger's $p=0.859$ and Begg's $p=0.834$ ), homozygous (Egger's $p=0.785$ and Begg's $p=0.452$ ), heterozygous (Egger's $p=0.667$ and Begg's $p=0.835$ ), recessive (Egger's $p=0.633$ and Begg's $p=0.929$ ), and dominant (Egger's $p=0.710$ and Begg's $p=0.881)$ models of TNF- $\alpha(-308 G>A)$ polymorphism.
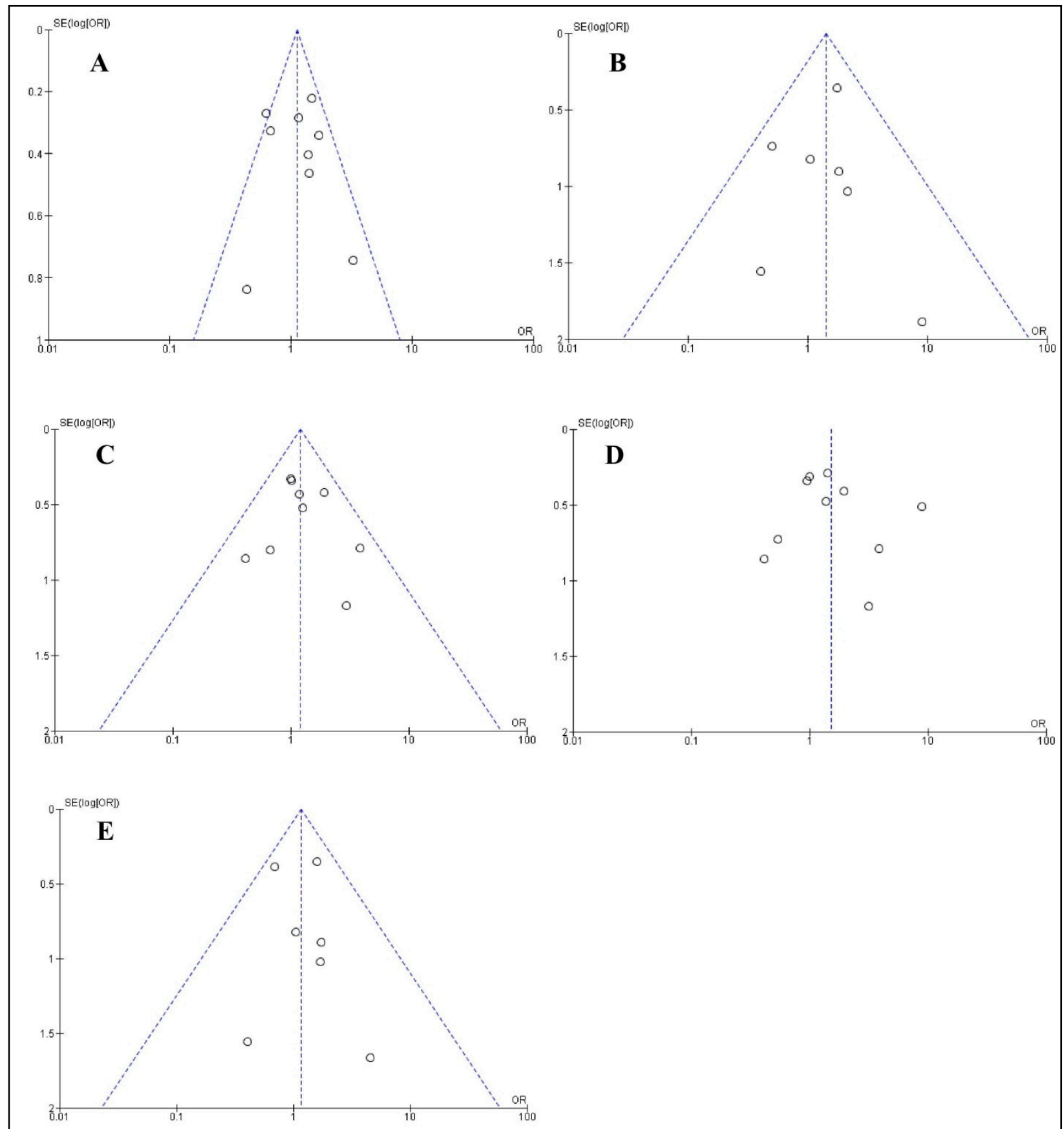

Figure 3. Funnel plot analyses of five genetic models for the association between TNF- $\alpha(-308 \mathrm{G}>A)$ polymorphism and peri-implant disease risk: (A) allelic, (B) homozygous, (C) heterozygous, (D) recessive, and (E) dominant. 


\section{Discussion}

Dental implants provide a great treatment option for patients with missing teeth by replacing the root of the tooth with fixed permanent artificial tooth roots that are implanted into the jawbone matching the natural ones and supporting the prosthetic crowns [21].

The main results of the present meta-analysis showed that TNF- $\alpha$ ( $-308 \mathrm{G}>A), I L-10$ $(-1082 A>G), I L-10(-819 C>T)$, and IL-10 $(-592 A>C)$ polymorphisms were not associated with PID risk. Out of TNF- $\alpha(-308 G>A), I L-10(-1082 A>G)$, and IL-10 $(-819 C>T)$ polymorphisms, the TSA confirmed the result of only IL-10 $(-819 C>T)$ polymorphism, indicating the need for more evidence on TNF- $\alpha(-308 G>A)$ and $I L-10$ $(-1082 A>G)$ polymorphisms. The TNF- $\alpha(-308 G>A)$ polymorphism had a significant elevated risk in Asian PID patients compared to controls. Moreover, the meta-regression confirmed that publication year and number of individuals were not confounding factors on the association between TNF- $\alpha(-308 G>A)$ polymorphism and PID susceptibility.

One research showed increased salivary TNF- $\alpha$ level in cases with peri-implant clinical condition, especially in patients with peri-implantitis [35]. Another research confirmed significantly higher serum level of TNF- $\alpha$ in peri-implantitis patients compared to controls, indicating the pivotal role of these cytokines in peri-implantitis [36]. Farhad et al. [37] concluded that IL-10 level increased in patients with PID compared to individuals with healthy peri-implant tissues, which was also confirmed by many other studies [38-40]. Differences in the level of two cytokines between PID patients and controls and the lack of association between the two polymorphisms and the risk of PID in our meta-analysis may indicate the influence of other genetic as well as environmental factors. Future studies might need to explore the influence of these factors.

A meta-analysis examined the association between smoking, radiotherapy, diabetes, and osteoporosis and the risk of dental implant failure [41]. Smoking [17,41-43] and radiotherapy [41] are considered the most significant risk factors for dental implant failure. It would be interesting to explore the role of these risk factors on the relationship between gene polymorphism and PID. However, we could not run a meta-regression analysis to assess the effect of these risk factors on the association between gene polymorphisms and PID risk due to unavailability of such data. Wilson and Nunn evaluated the effect of IL-1 polymorphism (smoking and age on dental implant failures) and found that smoking was the only strong risk factor for implant failure [44]. Feloutzis et al. observed similar findings suggesting that IL-1 genotype could further precipitate the detrimental effect of smoking on peri-implant bone loss [45]. Pathogenic bacteria, lack of oral hygiene, and alcohol consumption have also been reported as factors associated with peri-implantitis [42,43]. Research has also indicated the possible effect of systemic diseases on peri-implant bone loss, and most studies therefore recruit PID patients without any systemic diseases [46-49]. Most studies in our meta-analysis selected individuals who did not smoke or the smoking status was matched between two groups (patients and controls) $[14,18,27,28,30,32,33]$ and without any systemic disease in both cases and controls $[18,27,30,33]$.

Although research exploring the effect of several systemic, habitual, and clinical risk factors on the risk of PID is vast, the effect of genetic risk factors has not been well studied $[50,51]$. This meta-analyses evaluated TNF- $\alpha(-308 \mathrm{G}>A)$ and IL-10 $(-1082 \mathrm{~A}>\mathrm{G})$ polymorphisms [21] or TNF- $\alpha$ ( $-308 G>A$ ) polymorphism [3] alone, and no association was observed between any of these polymorphisms and the risk of PID disease. In our meta-analysis, there was an association between TNF- $\alpha(-308 \mathrm{G}>A$ ) polymorphism and PID in Asian patients. We need to further explore the role of ethnicity on the association of the mentioned polymorphisms and PID risk, especially TNF- $\alpha(-308 G>A)$ polymorphism.

This meta-analysis had several limitations, namely (1) few studies and lack of subgroup analysis for $I L-10$ polymorphisms, (2) smaller sample sizes in some of the included studies, (3) inclusion of smokers as cases and controls in some studies, and (4) the studies that included populations from Asian ethnicity were both from China, meaning the results might not be representative of all Asian population. Lack of publication bias, stability of 
the pooled data, and the confirmation of the pooled results by TSA would be the important strengths of this meta-analysis.

\section{Conclusions}

The pooled analysis of the present meta-analysis showed that TNF- $\alpha(-308 \mathrm{G}>A)$, IL-10 $(-1082 A>G), I L-10(-819 C>T)$, and IL-10 $(-592 A>C)$ polymorphisms were not associated with PID risk, whereas TNF- $\alpha(-308 G>A)$ polymorphism was associated with a significant elevated risk of PID in patients of Asian ethnicity.

Author Contributions: Conceptualization, S.K.T. and J.T.; methodology, S.K.T. and M.S.; formal analysis, M.S.; investigation, L.J.; resources, M.S. and J.T.; data curation, P.C., J.T. and M.S.; writingoriginal draft preparation, L.J. and P.C.; and writing-review and editing, L.J., S.K.T. and J.T. All authors have read and agreed to the published version of the manuscript.

Funding: No financial support was received for this study.

Institutional Review Board Statement: Not applicable.

Informed Consent Statement: Not applicable.

Data Availability Statement: The data that support the findings of this study are available on request from the corresponding author.

Acknowledgments: Santosh Kumar Tadakamadla acknowledges the support of National Health and Medical Research Council (Australia) through early career fellowship.

Conflicts of Interest: The authors have no conflict of interest regarding the publication of this study.

\section{References}

1. Van Steenberghe, D.; Klinge, B.; Lindén, U.; Quirynen, M.; Herrmann, I.; Garpland, C. Periodontal indices around natural and titanium abutments: A longitudinal multicenter study. J. Periodontol. 1993, 64, 538-541. [CrossRef]

2. Figuero, E.; Graziani, F.; Sanz, I.; Herrera, D.; Sanz, M. Management of peri-implant mucositis and peri-implantitis. Periodontology 2000 2014, 66, 255-273. [CrossRef]

3. Mo, Y.-Y.; Zeng, X.-T.; Weng, H.; Cen, Y.; Zhao, Q.; Wen, X. Association between tumor necrosis factor-alpha G-308A polymorphism and dental peri-implant disease risk: A meta-analysis. Medicine 2016, 95, e4425. [CrossRef]

4. Zitzmann, N.U.; Berglundh, T. Definition and prevalence of peri-implant diseases. J. Clin. Periodontol. 2008, 35, $286-291$. [CrossRef]

5. Jepsen, S.; Berglundh, T.; Genco, R.; Aass, A.M.; Demirel, K.; Derks, J.; Figuero, E.; Giovannoli, J.L.; Goldstein, M.; Lambert, F. Primary prevention of peri-implantitis: Managing peri-implant mucositis. J. Clin. Periodontol. 2015, 42, S152-S157. [CrossRef]

6. Singh, R.; Parihar, A.S.; Vaibhav, V.; Kumar, K.; Singh, R.; Jerry, J.J. A 10 years retrospective study of assessment of prevalence and risk factors of dental implants failures. J. Fam. Med. Prim. Care 2020, 9, 1617.

7. Cecchinato, D.; Parpaiola, A.; Lindhe, J. A cross-sectional study on the prevalence of marginal bone loss among implant patients. Clin. Oral Implant. Res. 2013, 24, 87-90. [CrossRef]

8. Moy, P.K.; Medina, D.; Shetty, V.; Aghaloo, T.L. Dental implant failure rates and associated risk factors. Int. J. Oral Maxillofac. Implant. 2005, 20, 569-577.

9. Kadkhodazadeh, M.; Tabari, Z.A.; Pourseyediyan, T.; Najafi, K.; Amid, R. Relationship between Genetic Polymorphisms with Periodontitis and Peri-Implantitis in the Iranian Population: A Literature Review. J. Long-Term Eff. Med Implant. 2016, 26, 183-190. [CrossRef]

10. Lee, S.; Kim, J.-Y.; Hwang, J.; Kim, S.; Lee, J.-H.; Han, D.-H. Investigation of pathogenic genes in peri-implantitis from implant clustering failure patients: A whole-exome sequencing pilot study. PLoS ONE 2014, 9, e99360. [CrossRef] [PubMed]

11. Van Dyke, T.E. The impact of genotypes and immune reactivity on peri-implant inflammation: Identification and therapeutic use of anti-inflammatory drugs and immunomodulators. Eur J Oral Implant. 2012, 5, S51-S60.

12. Cullinan, M.; Westerman, B.; Hamlet, S.; Palmer, J.; Faddy, M.; Seymour, G.; Middleton, P.; Taylor, J. Progression of periodontal disease and interleukin-10 gene polymorphism. J. Periodontal Res. 2008, 43, 328-333. [CrossRef]

13. Ding, C.; Ji, X.; Chen, X.; Xu, Y.; Zhong, L. TNF- $\alpha$ gene promoter polymorphisms contribute to periodontitis susceptibility: Evidence from 46 studies. J. Clin. Periodontol. 2014, 41, 748-759. [CrossRef] [PubMed]

14. He, K.; Jian, F.; He, T.; Tang, H.; Huang, B.; Wei, N. Analysis of the association of TNF- $\alpha$, IL-1A, and IL-1B polymorphisms with peri-implantitis in a Chinese non-smoking population. Clin. Oral Investig. 2020, 24, 693-699. [CrossRef]

15. Saremi, L.; Shafizadeh, M.; Esmaeilzadeh, E.; Ghaffari, M.E.; hosein Mahdavi, M.; Amid, R.; Kadkhodazadeh, M. Assessment of IL-10, IL-1ß and TNF- $\alpha$ gene polymorphisms in patients with peri-implantitis and healthy controls. Mol. Biol. Rep. 2021, 48, 2285-2290. [CrossRef] [PubMed] 
16. Ladeira Casado, P.; Villas-Boas, R.; de Mello, W.; Leite Duarte, M.E.; Mauro Granjeiro, J. Peri-implant disease and chronic periodontitis: Is interleukin-6 gene promoter polymorphism the common risk factor in a Brazilian population? Int. J. Oral Maxillofac. Implant. 2013, 28, 35-43. [CrossRef]

17. Petkovic-Curcin, A.; Zeljic, K.; Cikota-Aleksic, B.; Dakovic, D.; Tatic, Z.; Magic, Z. Association of Cytokine Gene Polymorphism with Peri-implantitis, Risk. Int. J. Oral Maxillofac. Implant. 2017, 32, e241-e248. [CrossRef]

18. Pigossi, S.C.; Alvim-Pereira, F.; Montes, C.C.; Finoti, L.S.; Secolin, R.; Trevilatto, P.C.; Scarel-Caminaga, R.M. Genetic association study between Interleukin 10 gene and dental implant loss. Arch. Oral Biol. 2012, 57, 1256-1263. [CrossRef]

19. Moore, K.W.; de Waal Malefyt, R.; Coffman, R.L.; O'Garra, A. Interleukin-10 and the interleukin-10 receptor. Annu. Rev. Immunol. 2001, 19, 683-765. [CrossRef]

20. Duarte, P.M.; De Mendonça, A.C.; Máximo, M.B.B.; Santos, V.R.; Bastos, M.F.; Nociti Júnior, F.H. Differential cytokine expressions affect the severity of peri-implant disease. Clin. Oral Implant. Res. 2009, 20, 514-520. [CrossRef]

21. Santiago Junior, J.F.; Biguetti, C.C.; Matsumoto, M.A.; Abu Halawa Kudo, G.; Parra da Silva, R.B.; Pinto Saraiva, P.; Fakhouri, W.D. Can genetic factors compromise the success of dental implants? A systematic review and meta-analysis. Genes 2018, 9, 444. [CrossRef] [PubMed]

22. Moher, D.; Liberati, A.; Tetzlaff, J.; Altman, D.G. Preferred reporting items for systematic reviews and meta-analyses: The PRISMA statement. Int. J. Surg 2010, 8, 336-341. [CrossRef] [PubMed]

23. DerSimonian, R.; Laird, N. Meta-analysis in clinical trials. Control. Clin. Trials 1986, 7, 177-188. [CrossRef]

24. Mantel, N.; Haenszel, W. Statistical aspects of the analysis of data from retrospective studies of disease. J. Natl. Cancer Inst. 1959, 22, 719-748. [PubMed]

25. Imberger, G.; Thorlund, K.; Gluud, C.; Wetterslev, J. False-positive findings in Cochrane meta-analyses with and without application of trial sequential analysis: An empirical review. BMJ Open 2016, 6, e011890. [CrossRef]

26. Wetterslev, J.; Jakobsen, J.C.; Gluud, C. Trial sequential analysis in systematic reviews with meta-analysis. BMC Med. Res. Methodol. 2017, 17, 1-18. [CrossRef]

27. Campos, M.I.G.; dos Santos, M.C.L.G.; Trevilatto, P.C.; Scarel-Caminaga, R.M.; Bezerra, F.J.B.; Line, S.R.P. Early failure of dental implants and TNF- $\alpha$ (G-308A) gene polymorphism. Implant Dent. 2004, 13, 95-101. [CrossRef]

28. Cury, P.R.; Horewicz, V.V.; Ferrari, D.S.; Brito, R., Jr.; Sendyk, W.R.; Duarte, P.M.; Shibli, J.A. Evaluation of the effect of tumor necrosis factor-alpha gene polymorphism on the risk of peri-implantitis: A case-control study. Int. J. Oral Maxillofac. Implant. 2009, 24, 1101-1105.

29. Lu, X.; Lin, Y.; Li, Z. The relationship between TNF-A-308 gene polymorphism and marginal bone loss around dental implants. Guangdong Periodontol 2009, 1006-5245.

30. Gurol, C.; Kazazoglu, E.; Dabakoglu, B.; Korachi, M. A Comparative Study of the Role of Cytokine Polymorphisms Interleukin-10 and Tumor Necrosis Factor Alpha in Susceptibility to Implant Failure and Chronic Periodontitis. Int. J. Oral Maxillofac. Implant. 2011, 26, 955-960.

31. Jacobi-Gresser, E.; Huesker, K.; Schütt, S. Genetic and immunological markers predict titanium implant failure: A retrospective study. Int. J. Oral Maxillofac. Surg. 2013, 42, 537-543. [CrossRef] [PubMed]

32. Rakic, M.; Petkovic-Curcin, A.; Struillou, X.; Matic, S.; Stamatovic, N.; Vojvodic, D. CD14 and TNF $\alpha$ single nucleotide polymorphisms are candidates for genetic biomarkers of peri-implantitis. Clin. Oral Investig. 2015, 19, 791-801. [CrossRef] [PubMed]

33. Ribeiro, R.; Melo, R.; Tortamano Neto, P.; Vajgel, A.; Souza, P.R.E.; Cimões, R. Polymorphisms of IL-10 (-1082) and RANKL (-438) genes and the failure of dental implants. Int. J. Dent. 2017, 2017, 1-6. [CrossRef]

34. Broker, R.d.C.; Doetzer, A.D.; de Souza, C.M.; Alvim-Pereira, F.; Alvim-Pereira, C.C.; Trevilatto, P.C. Clinical aspects and polymorphisms in the LTA, TNFA, LTB genes and association with dental implant loss. Clin. Implant Dent. Relat. Res. 2018, 20, 954-961. [CrossRef] [PubMed]

35. Gomes, A.M.; Douglas-de-Oliveira, D.W.; Ferreira, S.D.; Silva, T.A.D.; Cota, L.O.M.; Costa, F.O. Periodontal disease, peri-implant disease and levels of salivary biomarkers IL-1 $\beta$, IL-10, RANK, OPG, MMP-2, TGF- $\beta$ and TNF- $\alpha$ : Follow-up over 5 years. J. Appl. Oral Sci. 2019, 27. [CrossRef] [PubMed]

36. Darabi, E.; Kadkhoda, Z.; Amirzargar, A. Comparison of the levels of tumor necrosis factor- $\alpha$ and interleukin-17 in gingival crevicular fluid of patients with peri-implantitis and a control group with healthy implants. Iran. J. Allergy Asthma Immunol. 2013, 12, 75-80.

37. Farhad, S.Z.; Rezazadeh, F.; Mohammadi, M. Interleukin-17 and interleukin-10 as inflammatory and prevention biomarkers in periimplant diseases. Int. J. Prev. Med. 2019, 10, 137. [CrossRef]

38. Ata-Ali, J.; Flichy-Fernández, A.J.; Alegre-Domingo, T.; Ata-Ali, F.; Palacio, J.; Peñarrocha-Diago, M. Clinical, microbiological, and immunological aspects of healthy versus peri-implantitis tissue in full arch reconstruction patients: A prospective cross-sectional study. BMC Oral Health 2015, 15, 1-10. [CrossRef]

39. Casado, P.L.; Canullo, L.; de Almeida Filardy, A.; Granjeiro, J.M.; Barboza, E.P.; Duarte, M.E.L. Interleukins $1 \beta$ and 10 expressions in the periimplant crevicular fluid from patients with untreated periimplant disease. Implant Dent. 2013, 22, 143-150. [CrossRef] [PubMed]

40. Ghighi, M.; Llorens, A.; Baroukh, B.; Chaussain, C.; Bouchard, P.; Gosset, M. Differences between inflammatory and catabolic mediators of peri-implantitis and periodontitis lesions following initial mechanical therapy: An exploratory study. J. Periodontal Res. 2018, 53, 29-39. [CrossRef] 
41. Chen, H.; Liu, N.; Xu, X.; Qu, X.; Lu, E. Smoking, radiotherapy, diabetes and osteoporosis as risk factors for dental implant failure: A meta-analysis. PLOS ONE 2013, 8, e71955. [CrossRef]

42. Galindo-Moreno, P.; Fauri, M.; Ávila-Ortiz, G.; Fernández-Barbero, J.E.; Cabrera-León, A.; Sánchez-Fernández, E. Influence of alcohol and tobacco habits on peri-implant marginal bone loss: A prospective study. Clin. Oral Implant. Res. 2005, 16, 579-586. [CrossRef]

43. Lindhe, J.; Meyle, J.; Periodontology, G.D.o.t.E.W.o. Peri-implant diseases: Consensus report of the sixth European workshop on periodontology. J. Clin. Periodontol. 2008, 35, 282-285. [CrossRef] [PubMed]

44. Wilson, T.G., Jr.; Nunn, M. The Relationship between the Interleukin-1 Periodontal Genotype and Implant Loss. Initial Data. J. Periodontol. 1999, 70, 724-729. [CrossRef] [PubMed]

45. Feloutzis, A.; Lang, N.P.; Tonetti, M.S.; Bürgin, W.; Brägger, U.; Buser, D.; Duff, G.W.; Kornman, K.S. IL-1 gene polymorphism and smoking as risk factors for peri-implant bone loss in a well-maintained population. Clin. Oral Implant. Res. 2003, 14, 10-17. [CrossRef] [PubMed]

46. Alvim-Pereira, F.; Montes, C.C.; Thomé, G.; Olandoski, M.; Trevilatto, P.C. Analysis of association of clinical aspects and vitamin D receptor gene polymorphism with dental implant loss. Clin. Oral Implant. Res. 2008, 19, 786-795. [CrossRef] [PubMed]

47. Costa-Junior, F.; Alvim-Pereira, C.; Alvim-Pereira, F.; Trevilatto, P.; de Souza, A.; Santos, M.C.L. Influence of MMP-8 promoter polymorphism in early osseointegrated implant failure. Clin. Oral Investig. 2013, 17, 311-316. [CrossRef] [PubMed]

48. Dos Santos, M.C.L.G.; Campos, M.I.G.; Souza, A.P.; Scarel-Caminaga, R.M.; Mazzonetto, R.; Line, S.R.P. Analysis of the transforming growth factor- $\beta 1$ gene promoter polymorphisms in early osseointegrated implant failure. Implant Dent. 2004, 13, 262-269. [CrossRef]

49. Vaz, P.; Gallas, M.; Braga, A.; Sampaio-Fernandes, J.; Felino, A.; Tavares, P. IL1 gene polymorphisms and unsuccessful dental implants. Clin. Oral Implant. Res. 2012, 23, 1404-1413. [CrossRef] [PubMed]

50. De Souza Batista, V.E.; Junior, J.F.S.; de Faria Almeida, D.A.; de Toledo Piza Lopes, L.F.; Verri, F.R.; Pellizzer, E.P. The effect of offset implant configuration on bone stress distribution: A systematic review. J. Prosthodont. 2015, 24, 93-99. [CrossRef] [PubMed]

51. Lemos, C.A.A.; de Souza Batista, V.E.; de Faria Almeida, D.A.; Júnior, J.F.S.; Verri, F.R.; Pellizzer, E.P. Evaluation of cement-retained versus screw-retained implant-supported restorations for marginal bone loss: A systematic review and meta-analysis. J. Prosthet. Dent. 2016, 115, 419-427. [CrossRef] [PubMed] 